\title{
Frontières
}

\section{Memento moris, vanités et natures mortes}

\section{Alain Paiement}

Volume 14, numéro 2, printemps 2002

La mort prononcée

URI : https://id.erudit.org/iderudit/1073981ar

DOI : https://doi.org/10.7202/1073981ar

Aller au sommaire du numéro

Éditeur(s)

Université du Québec à Montréal

ISSN

1180-3479 (imprimé)

1916-0976 (numérique)

Découvrir la revue

Citer ce document

Paiement, A. (2002). Memento moris, vanités et natures mortes. Frontières, 14(2), 91-92. https://doi.org/10.7202/1073981ar

Ce document est protégé par la loi sur le droit d'auteur. L'utilisation des services d'Érudit (y compris la reproduction) est assujettie à sa politique d'utilisation que vous pouvez consulter en ligne.

https://apropos.erudit.org/fr/usagers/politique-dutilisation/ 


\section{MEMENTO MORIS, VANITESS ET NATURES MORTES}

\section{Alain Paiement, \\ artiste et chargé de cours, Université Concordia, UQÀM.}

Nous avons choisi pour ce numéro, à partir de l'image de la Mort dans les cartes du tarot (p. 14), plusieurs images représentant des personnages squelettiques qui prennent des allures aussi multiples que les contextes d'où ils proviennent. Ces figures deviennent des memento moris, des spectres, des personnages carnavalesques ou des vanités. En alternance avec ces squelettes, nous avons des images de natures mortes. Genre pictural paradoxal dont la tradition remonte à l'Antiquité, la nature morte est principalement composée d'objets ou d'êtres inanimés, principalement des végétaux coupés et des animaux morts. La durée de vie de ces compositions éphémères se voit prolongée par celle de l'image. Lorsque celle-ci est de nature photographique, en considérant la très relative permanence de cette image, la finitude apparente des objets représentés n'est finalement retardée que pour une période plutôt brève à l'égard du désir de durée.

On dit nature morte en français et still life en anglais. Retraduisons. Dead nature, vie arrêtée. Still comme un arrêt instantané. Sinon, still life comme encore en vie. « Nature morte» est le nom d'une mort prononcée par défaut, par l'immortalisation d'un état du vivant avant qu'il ne pourrisse. Photographier ou peindre des fleurs et des feuilles permet effectivement au regard futur de voir encore vivant ce qui aura disparu.

\section{SQUELETTES}

Parmi les reproductions choisies, il y a deux images produites à Berlin en 19321933 durant la période de tourmente qui voit la montée du pouvoir nazi. Les artistes Otto Dix (Les sept péchés capitaux, p. 21) et Erwin Blumenfeld (Totenschädel, p. 10) désignent la politique pour le moins morbide du nouveau régime par leur utili- sation de la figure squelettique, annonciatrice d'une mort imminente. Ces images font écho aux fameux calaveras, les squelettes dessinés par l'artiste mexicain Jose Guadaloupe Posada (p. 67, 80) à la fin du XIX et au début du $\mathrm{XX}^{\mathrm{e}}$ siècle. À cette époque, l'artiste est caricaturiste pour de nombreux journaux d'opposition au gouvernement de plus en plus dictatorial de son pays. Apparaissent alors les premiers squelettes dont Posada fera les personnages principaux de nombreuses gravures. Ces calaveras peuvent danser, boire, se battre ou encore se pavaner. Ce ne sont pas seulement des représentations des pauvres, de l'injustice ou de la vanité des puissants; c'est l'ensemble du peuple mexicain qui se voit interprété par ces figures de la mort. Les dessins de couverture de revues et journaux qu'il a produits par centaines seront autant de critiques de la société mexicaine sous la dictature.

Lucie Robert (p. 3, 6, 71) dessine des personnages immobiles, à la fois charnus et décharnés, des corps presque sans opacité, dont la structure du squelette est souvent cousue à même le papier, discrètement. Au travers de tout leur corps circulent des flux d'encre et d'énergie innommée.

Alors que les figures du tarot ont une signification plutôt emblématique de la mort annoncée, les figures de Lucie Robert, par la gestuelle et la liquidité particulière avec lesquelles elles apparaissent, deviennent des images spectrales évoquant un état de changement.

Lionel Blanchard (p. 74) griffonne un squelette au stylo à bille sur une image d'homme nu, dont la pose évoque l'offre sexuelle. L'image provient d'un magazine de pornographie homosexuelle dans laquelle les corps des jeunes hommes en érection sont donnés à voir comme des objets de fantasme. Le dessin de l'artiste transforme ces objets avec une distance critique, sinon un certain cynisme à l'égard de la dimension futile des images. Dans la perspective non dite de la menace du sida, cette image devient alors un memento mori contemporain.

Vanité et reflets. À Vienne, dans une chapelle catholique et baroque, un autel ornementé (p. 29). Dorures et volutes. Marbres. Verre et orfèvrerie. La face de l'autel, visible par l'assemblée, est transparente. Une paroi de verre nous révèle l'intérieur: un squelette-reliquaire y est couché, tourné vers le chœur. Il nous regarde. « Rappelle-toi que tu meurs»: l'autel est un memento mori durant toutes les cérémonies qui s'y tiennent. À la suite de cette image, nous avons juxtaposé une photographie d'étalage muséal conçu par le sculpteur contemporain Donald Judd (p. 30) pour conserver une fantaisie baroque en porcelaine, qui se trouve dans le Musée des arts décoratifs de Vienne. Cette mise sous verre recèle une parenté formelle avec celle qui précède. Par ailleurs, elle poursuit et actualise la tradition de la nature morte.

\section{NATURES MORTES}

De Nicolas Baier (p. 59), nous avons juxtaposé une image de plante vivante qu'il avait envoyée comme invitation à une exposition de son travail, avec une image de la même plante, morte cette fois-ci, que l'on voyait en entrant dans la galerie. Une mort annoncée. Le traitement par facettes et fragmentation des deux images leur donne un aspect transformable, comme si la photographie que l'on voit n'était qu'un moment d'arrêt en cours de traitement informatique. L'instantané d'un processus de disparition (p. 61).

Roberto Pellegrinuzzi photographie de manière analytique une toute petite feuille d'arbre avant de la transposer par un assemblage d'impressions sur papier de riz, établissant alors une corrélation de nature analogique entre la surface du végétal et celle de l'empreinte photosensible (p. 83). La 
fragilité des deux est intrinsèquement liée. On a souvent désigné l'arrêt du temps photographique comme un arrêt de mort. Comment interpréter alors ce grand montage de photographies lumineuses aux couleurs saturées d'un bouquet de fleurs luxuriantes installé dans un centre hospitalier (p. 88), dont le flamboiement nous semble cousin de ces bouquets opulents de la peinture flamande ou hollandaise du XVII ${ }^{\mathrm{e}}$ siècle? Cette dimension mortifère de la photographie se voit aussi bien dans la série des écorchés, ces montages dans lesquels les visages auscultés en macro-photographie deviennent des masques mortuaires (p. 26, 27).
Une autre feuille (p. 49) : un calligramme islamique du XVII ${ }^{\mathrm{e}}$ siècle fait de dorure très finement appliquée sur une feuille de tabac, surlignant en or les nervures de la feuille, comme une autre écriture en dialogue avec l'arabe. Nature morte minimale conservant pour des siècles un extrait complexe de nature et de culture. Mémoire délicate.

D'autres fleurs, au XIX ${ }^{\mathrm{e}}$ siècle : des fleurs dans un vase, peintes avec réalisme par Fantin-Latour (p. 35), des fleurs jetées sur une surface blanche et photographiées par un inconnu vers 1870 (p. 36). Puis, une photographie de August Kotzch à la même époque, montrant simplement des patates dans une assiette (p. 94). Leurs formes diffèrent quelque peu de nos patates contemporaines, aux allures beaucoup plus régulières après un siècle de transformations génétiques. Image-témoin de presque rien qui change.

L'empreinte photographique désigne la mort de cet instant qu'elle imprime. Nous recherchons en vain la durée de son effet, substituant l'apparence imprimée à la réalité éphémère. En cela, elle nous apparaît toujours comme une mort prononcée.



